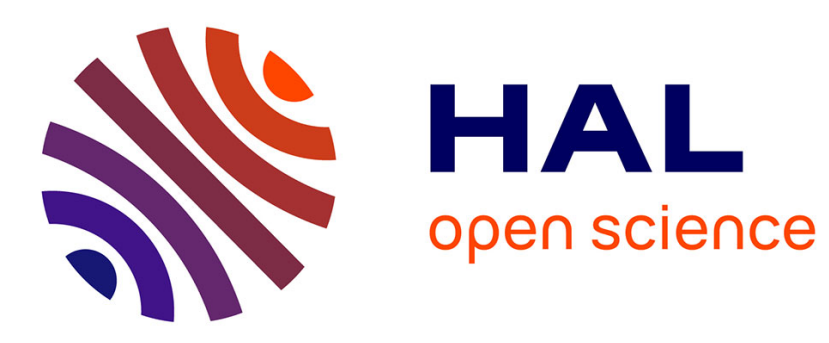

\title{
Filter Shape Index Modulation
}

Majed Saad, Jacques Palicot, Faouzi Bader, Ali Chamas Al Ghouwayel, Hussein Hijazi

\section{To cite this version:}

Majed Saad, Jacques Palicot, Faouzi Bader, Ali Chamas Al Ghouwayel, Hussein Hijazi. Filter Shape Index Modulation. 2021 IEEE International Conference on Communications (ICC), Jun 2021, Montreal (virtual), Canada. hal-03140371

\section{HAL Id: hal-03140371 https://hal.science/hal-03140371}

Submitted on 12 Feb 2021

HAL is a multi-disciplinary open access archive for the deposit and dissemination of scientific research documents, whether they are published or not. The documents may come from teaching and research institutions in France or abroad, or from public or private research centers.
L'archive ouverte pluridisciplinaire HAL, est destinée au dépôt et à la diffusion de documents scientifiques de niveau recherche, publiés ou non, émanant des établissements d'enseignement et de recherche français ou étrangers, des laboratoires publics ou privés. 


\title{
Filter Shape Index Modulation
}

\author{
Majed Saad ${ }^{\dagger}$, Jacques Palicot ${ }^{\dagger}$, Faouzi Bader*†, Ali Chamas Al Ghouwayel ${ }^{\ddagger}$, and Hussein Hijazi ${ }^{\S}$ \\ ${ }^{\dagger}$ IETR/CentraleSupélec, Rennes Campus, 35510 Cesson-Sévigné, France \\ *Institut Supérieur d'Electronique de Paris, 75006 Paris, France \\ ${ }^{\ddagger}$ EFREI Paris, 94800 Villejuif, France

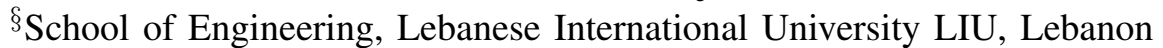 \\ majed.saad@ieee.org, jacques.palicot@centralesupelec.fr, faouzi.bader@isep.fr, ali.ghouwayel@efrei.fr \\ hussein.hijazi@liu.edu.lb
}

\begin{abstract}
Index Modulation (IM) techniques enable a significant Spectral Energy (SE) and/or Energy Efficiency (EE) enhancement. In this paper, a novel Filter IM domain is being explored, where a novel scheme Filter Shape Index Modulation (FSIM) is proposed. In contrast to most existing Single-Input Single-Output (SISO) IM systems, the proposed scheme achieves a higher SE/EE gain since it exploits all available time and frequency resources. The FSIM system transmits any conventional modulation (QAM, PSK,...) while conveying additional information bits by the index of pulse shaping filter shape. The optimal joint Maximum Likelihood detector is presented, and a low complexity optimal detector is proposed. In addition, an Inter-Symbol Interference (ISI) estimation and cancellation technique is developed to enable the usage of non-Nyquist filters. Finally, the results reveal that FSIM has a similar performance with the two proposed detectors, and it achieves an important performance gain of up to $6 \mathrm{~dB}$ compared to equivalent schemes of the same SE in AWGN and frequency selective channels.

Index Terms-Index Modulation (IM), Filter Index Modulation domain, Filter Domain (FD), Filter Shape Index Modulation (FSIM), pulse-shaping filter, maximum likelihood, matched filter detector, spectral efficiency, energy efficiency, SISO
\end{abstract}

\section{INTRODUCTION}

Beyond 5G wireless communication intends to reach an ultra-high throughput and/or reduce the energy consumption, where these requirements differ among the use-cases. Recently, Index Modulation (IM) evolution shows its ability to knock out the competition on achieving higher Spectral Efficiency (SE) and/or Energy Efficiency (EE) limits. The basic idea of IM is the transmission of implicit information bits by indexing an element (time slot, frequency band, transmit or receive antenna,...) that the receiver can detect. For instance, the time, frequency, and spatial IM domain have been explored separately and jointly. In the SISO context, the time/frequency IM dimensions activate one or more time slots/frequency bands (sub-carriers) to convey the Virtual Bits (VBs). However, these schemes sacrifice in some available time/frequency resources to enable the indexation, and thus their SE is penalized, especially with high order modulations. Indeed, this limited SE enhancement and even loss comes with an important EE gain, which is suitable for low power Internetof-Things (IoT) devices.

In the last decade, different schemes of time/frequency IM domains appeared, where the Pulse Position Modulation
(PPM) [1] can be considered as the first modulation using only the time-IM. Afterward, a Single Carrier (SC) with time slots IM (SC-TIM) allowed the activation of more than one time slots while transmitting an Amplitude-Phase Modulation (APM) symbol [2], similar to the previously proposed frequency/Sub-carrier IM (SIM) counterparts OFDM-SIM [3], [4]. The latter is upgraded to achieve higher SE by using either Quadrature OFDM-SIM with different indexation on the inphase (I)/quadrature (Q) components, or the OFDM-Variable SIM (OFDM-VSIM) with a variable number of activated subcarriers [5]. For the same reason, the time IM and frequency IM are introduced with the non-orthogonal system like Fasterthan-Nyquist SC-FTN-IM [6], and Spectrally efficient Frequency Division Multiplexing (SeFDM)-IM [7], respectively. Moreover, the modulation type IM that conveys information by the selected APM is combined with SC-TIM and OFDM-IM to generate their Dual Mode (DM) version DM-SC-IM [8], DMOFDM-IM [9]. With the help of modulation type IM, these DM schemes overcome the SE limitation in time/frequency IM by transmitting another distinguishable APM on the nonactivated time slot(s)/frequency band(s).

The spatial IM domain is explored to enhance the SE/EE, where its combination with low order modulations is a promising candidate for ultra-high data rates with low power consumption [10], [11]. However, the spatial IM domain suffers from important degradation due to spatial correlation that cannot be completely compensated, especially in highly correlated channel like in sub-Terahertz bands [12]. In this paper, a novel IM domain, called Filter IM domain, is explored by proposing "Filter Shape Index Modulation" (FSIM) scheme motivated by the advantage of IM combination with low order SC schemes for ultra-high data rate with low power consumption. The proposed FSIM scheme conveys information by an APM symbol and an index of a filter shape. In addition, FSIM provides significant EE gain and it allows achieving the highest SE gain in SISO and MIMO systems by changing the indexed pulse shaping filter at the symbol rate and exploiting all available time/frequency resources.

The rest of the paper is organized as follows. The novel FSIM transceiver is presented in section II, where different detectors are used, and an ISI cancellation technique is proposed. A comparison with widely used existing equivalent schemes 
is presented in section III. Finally, section IV concludes the paper.

The notations are as follows: Bold lower case is used for vectors. The characters $*$ and $\star$ denotes the convolution and cross correlation respectively. $\mathcal{C N}\left(\mu, \sigma^{2}\right)$ denotes the complex normal distribution of a random variable having mean $\mu$ and variance $\sigma^{2} .\lfloor x\rfloor(\lceil x\rceil)$ denotes the floor (ceil) function that means the largest (smallest) integer less (greater) than or equal to $x$. $\|$.$\| stands for the Frobenius norm. \mathbb{E}[$.$] denotes the$ expectation.

\section{PRoposed FSIM System ModeL}

\section{A. FSIM Transmitter}

The proposed system considers a SISO transmission mode, where the binary source information sequence $b$ is divided into two streams $b_{1}$ and $b_{2}$ as shown in Fig. 1. Bit-stream $b_{1}$ is mapped by $M$-ary APM, such as QAM or PSK, etc. The bit-stream $b_{2}$ is encapsulated in the index $i$ of the selected filter. The selected filter $\mathbf{f}_{i}[m]$ of length $L$ samples is then used as a pulse-shaping filter where $m$ is the sample index taking values between 0 and $L-1$. The filter is truncated in the time domain to $\eta$ APM symbols and sampled at a rate of $\lambda$ samples per symbol, yielding $L=\eta \cdot \lambda+1$ samples. $L$ is restricted to odd in order to have a linear phase filter without a half-sample period shift.

We assume that there is a filter bank with $N$ distinguishable filters as shown in Fig. 1 ( $N$ is a power of 2$)$. Hence, the number of bits per FSIM symbol $b_{\text {FSIM }}$ can be expressed as:

$$
b_{\mathrm{FSIM}}=\log _{2}(N)+\log _{2}(M) .
$$

Thus, SE is enhanced by $\log _{2}(N)$ over conventional $M$-ary APM systems thanks to filter indexation at each symbol period.

The output of the filter bank for the $n$-th APM complex symbol $c_{n}$ is denoted by signals $s_{n}[m]$ and given as follows:

$$
s_{n}[m]=\left(f_{i_{n}} * c_{n}^{\prime}\right)[m]=c_{n} f_{i_{n}}[m],
$$

where $c_{n}^{\prime}$ is an up-sampled version of $c_{n}$ with factor $L$ and $i_{n} \in\{0,1, \ldots, N-1\}$ is the filter index selected for the $n^{t h}$ symbol based on the bit-stream $b_{2}$.

The signals $s_{n}[\mathrm{~m}]$ are then passed through the Overlap-Add (OLA) block as shown in Fig. 1 to generate $\lambda$ samples for each APM symbol. The $\lambda$ desired samples for the $n^{\text {th }}$ APM symbol $c_{n}$ are given by $x_{n}[p]$ as follows:

$$
x_{n}[p]=\sum_{n^{\prime}=n-\lceil\eta / 2\rceil}^{n+\lfloor\eta / 2\rfloor} s_{n^{\prime}}\left[p-\left(n^{\prime}-n\right) \lambda\right],
$$

where the index $p=p_{\text {center }}-\lceil\lambda / 2\rceil+1, \ldots, p_{\text {center }}+\lfloor\lambda / 2\rfloor$ and the index of the middle desired sample is $p_{\text {center }}=\frac{L-1}{2}$.

Equation (3) can be rewritten in terms of interference as:

$$
\mathbf{x}_{n}[p]=\mathbf{I}_{\lceil\eta / 2\rceil}^{n-\lceil\eta / 2\rceil}+\ldots+\mathbf{I}_{1}^{n-1}+\mathbf{s}_{n}+\mathbf{I}_{-1}^{n+1}+\ldots+\mathbf{I}_{-\lfloor\eta / 2\rfloor}^{n+\lfloor\eta / 2\rfloor} .
$$

Note that a conventional transceiver with any $M$-ary APM schemes can be considered a special case of our proposed system where all the filters in the bank are all replaced by the same Nyquist pulse-shaping filter, e.g. Root-Raised Cosine (RRC). In other words, the proposed system is equivalent to a conventional transceiver when using the same Nyquist filter for all symbols. Finally, a frame $\mathbf{x}_{f}$ is created by appending the $N_{s}$ FSIM symbols with $N_{Z P}$ zero-prefix symbols $\left(\lambda N_{Z P}\right.$ samples) $\left(\mathbf{x}_{f}=\left[0_{1}, \ldots, 0_{N_{Z P}}, \mathbf{x}_{-\lfloor\eta / 2\rfloor}, \ldots, \mathbf{x}_{\left\lceil N_{s}+\eta / 2\right\rceil}\right]\right)$.

\section{B. FSIM Receiver}

The receiver scheme proposed by our approach is represented in Fig. 1, where the received signal $y$ is expressed in the time domain as:

$$
y(t)=\left(h * x_{f}\right)(t)+v(t),
$$

where $h(t)$ is the impulse response of a multipath frequencyselective fading channel with $J$ paths and maximum delay spread of $(J-1) T_{\text {sym }}$, where $T_{\text {sym }}=\lambda . T_{s}$ is the APM symbol period and $T_{s}$ is the sampling period. Note that $N_{Z P} \geq(J-$ 1 ) in order to avoid the inter-frame interference. $v(t)$ is an Additive White Gaussian Noise (AWGN) with zero mean and variance $\sigma_{v}^{2}$, i.e, $\mathcal{C N}\left(0, \sigma_{v}^{2}\right)$. The power of transmitted symbols $c_{n}$ and the used filters $f_{i_{n}}$ are normalized: $\mathbb{E}\left[\left\|c_{n}\right\|\right]=1$ and $\left\|\mathbf{f}_{i}\right\|^{2}=\sum_{m=0}^{L-1} f_{i}^{2}[m]=1$ respectively.

First, the receiver compensates for fading channel distortion on all received samples containing implicit information from the selected filter index. For each received frame, the zeroprefix is eliminated, then a linear equalizer like Minimum Mean-Square Error-FDE (MMSE-FDE) [13] is used to mitigate the channel effect and recover the signal $\hat{x}$ as depicted in Fig. 1.

Afterwards, the equalized signal $\hat{x}$ is passed to the ISI estimation and cancellation that removes the controlled ISI from the filter shapes used. ISI cancellation is required if the filters used do not meet the Nyquist ISI criterion. The condition for zero ISI in the AWGN channel is:

$$
\left(f_{i} * g_{i}\right)\left(n . T_{\text {sym }}\right)= \begin{cases}1 & n=0 \\ 0 & n \neq 0\end{cases}
$$

for any integer $n$ and $i=1, \ldots, N$, where $g_{i}$ is the impulse response of the MF. Note that when this condition is satisfied, only one pulse has an effect at an integer multiple of the symbol period. However, this condition is relaxed in the proposed system when the target is to enhance the SE of $M$ ary APM, and then ISI will occur naturally. Therefore, The ISI cancellation process is required before detecting the APM symbol and selected filter index. The APM symbol and index detection can be performed jointly using a joint ML detector or separately using an MF detector. The The proposed detectors are described below.

1) Joint ML Detector: The equalized signal $\hat{x}$ is fed to the ISI cancellation block, which aims to regenerate the free-ISI signal $\hat{\mathbf{s}}$ composed of each of the $L$ samples. These signals $\hat{\mathbf{s}}$ are then fed to the detector to jointly recover the filter index and transmitted symbols. Detection can be performed using an ML detector that does an exhaustive search for all possible APM symbols and filters $\mathbf{f}_{i}$ combinations as follows:

$$
\{\hat{\imath}, \hat{c}\}=\arg \min _{\mathbf{f}_{i} \in \psi, c \in \chi}\left\|\hat{\mathbf{s}}-\mathbf{f}_{i} * c\right\|^{2}
$$




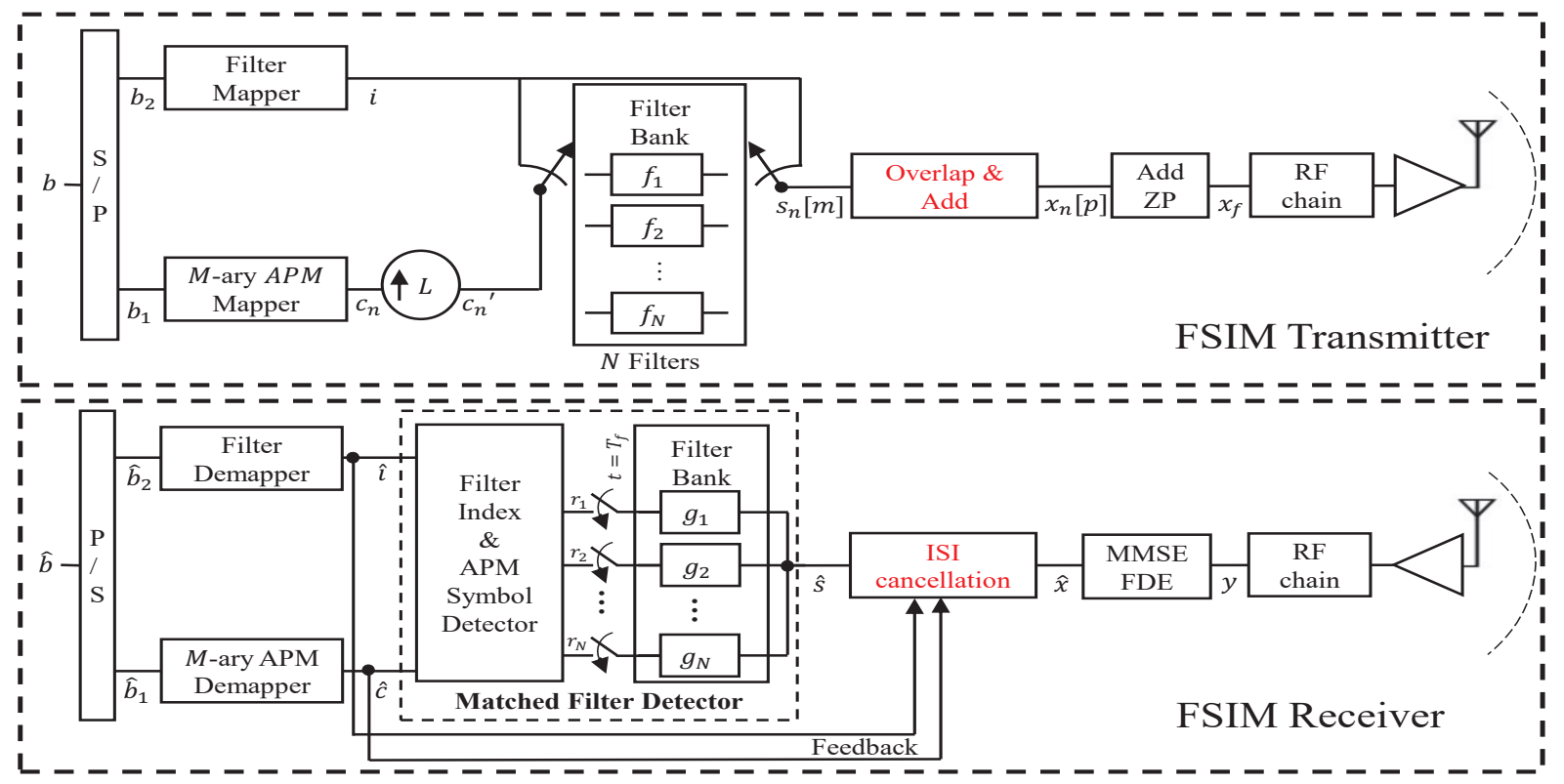

Fig. 1: System model of the FSIM-based transmitter and receiver with MF-based detector using $N$ filters of length $L$ and $M$-ary APM. Note that the detector can be replaced by the joint ML detector.

where $\psi$ and $\chi$ denote the set of $N$ filter's shapes and the $M$ ary APM constellation, respectively. $\hat{\imath}$ and $\hat{c}$ are the estimated filter indices of $\mathbf{f}_{i}$ and the estimated APM symbol, respectively. Such a receiver's system model is similar to that illustrated in Fig. 1 but by replacing the MF-based detector with an ML detector.

2) Matched Filter-based Detector: Detection of the selected filter index can be performed using a bank of MFs or a bank of correlators. This paper will consider the MFbased detector, knowing that both detectors provide the same results. Figure 1 depicts the system model where detection is performed after ISI cancellation. The detector consists of a $N$ matched filter $g_{k}$ where $g_{k}(t)=f_{k}\left(T_{f}-t\right)$ with $0 \leq t \leq T_{f}$, $T_{f}=L \cdot T_{s}$. The outputs of these filters $r_{k}(t)$ are given by:

$$
\begin{aligned}
r_{k}(t) & =\int_{0}^{t} \hat{s}(\tau) g_{k}(t-\tau) d \tau, \quad k=1,2, \ldots, N \\
& =\int_{0}^{t} \hat{s}(\tau) f_{k}\left(T_{f}-t+\tau\right) d \tau .
\end{aligned}
$$

These filter outputs are evaluated at the instant $T_{f}$

$$
r_{k}=\int_{0}^{T_{f}} \hat{s}(\tau) f_{k}(\tau) d \tau, \quad k=1,2, \ldots, N .
$$

Thus, the decision on the selected filter index, being used at the transmitter, is based on selecting the largest energy of the sampled MF outputs as follows:

$$
\hat{\imath}=\arg \max _{k}\left\|r_{k}\right\|^{2} \text {. }
$$

Once the filter index is detected, $r_{\hat{\imath}}$ is decoded by using an APM detector that determines the ML transmitted symbol $\hat{c}$ in the constellation set $\chi$. Note that any other APM detector can be used. Finally, the detected APM symbol $\hat{c}$ and the filter index $\hat{\imath}$ are sent to the corresponding demappers to deduce the bit-stream $\hat{b}_{1}$ and $\hat{b}_{2}$, respectively.
3) ISI Cancellation: FSIM's principle is to convey additional bits of information through the index of the transmission filter being selected at the transmitter. Thus, the success of this modulation is based on the ability to detect correctly at the first stage, the index of the selected filter $f_{i}$. For this reason, the filter bank cannot contain only Nyquist filters due to the inherent high cross-correlation between possible Nyquist filters. One of the solutions that could allow the detection of the correlated Nyquist filter is to use the same filter shape for many successive symbols. However, this solution contradicts the FSIM approach, aiming to increase SE in contrast to other SISO-IM schemes. Therefore, the filter bank used in this modulation is expected to generate ISI but predictable depending on the filter shapes.

Considering an AWGN channel or a perfect channel equalization, the received signal can be written as follows:

$$
\begin{aligned}
y(t) & =x(t)+v(t)=\sum_{n} s_{n}\left(t-n \cdot T_{\text {sym }}\right)+v(t) \\
& =\sum_{n} c_{n} f_{i_{n}}\left(t-n \cdot T_{\text {sym }}\right)+v(t) .
\end{aligned}
$$

This signal is sampled at the rate of $T_{s}$ and the $L$ received samples corresponding to APM symbol $c_{n}$ is given as follows:

$$
\begin{aligned}
y_{n}[m]= & (c_{n} \cdot f_{i_{n}}[m]+\underbrace{\sum_{n^{\prime}>n} c_{n^{\prime} \cdot} \cdot f_{i_{n^{\prime}}}\left[m-\left(n^{\prime}-n\right) \lambda\right]}_{\text {anticausal ISI }} \\
& +\underbrace{\sum_{n^{\prime}<n} c_{n^{\prime}} \cdot f_{i_{n^{\prime}}}\left[m-\left(n^{\prime}-n\right) \lambda\right]}_{\text {causal }})+v_{n}[m] \\
= & c_{n} . f_{i_{n}}[m]+I S I_{n}[m]+v_{n}[m],
\end{aligned}
$$

where $f_{i_{n}}$ represents the $i^{\text {th }}$ filter used to transmit the $n^{\text {th }}$ symbol and $v_{n}[m]$ is the noise sampled on $t=n \cdot T_{\text {sym }}+m \cdot T_{s}$. Note that if the used pulse-shaping filters satisfy Nyquist's 


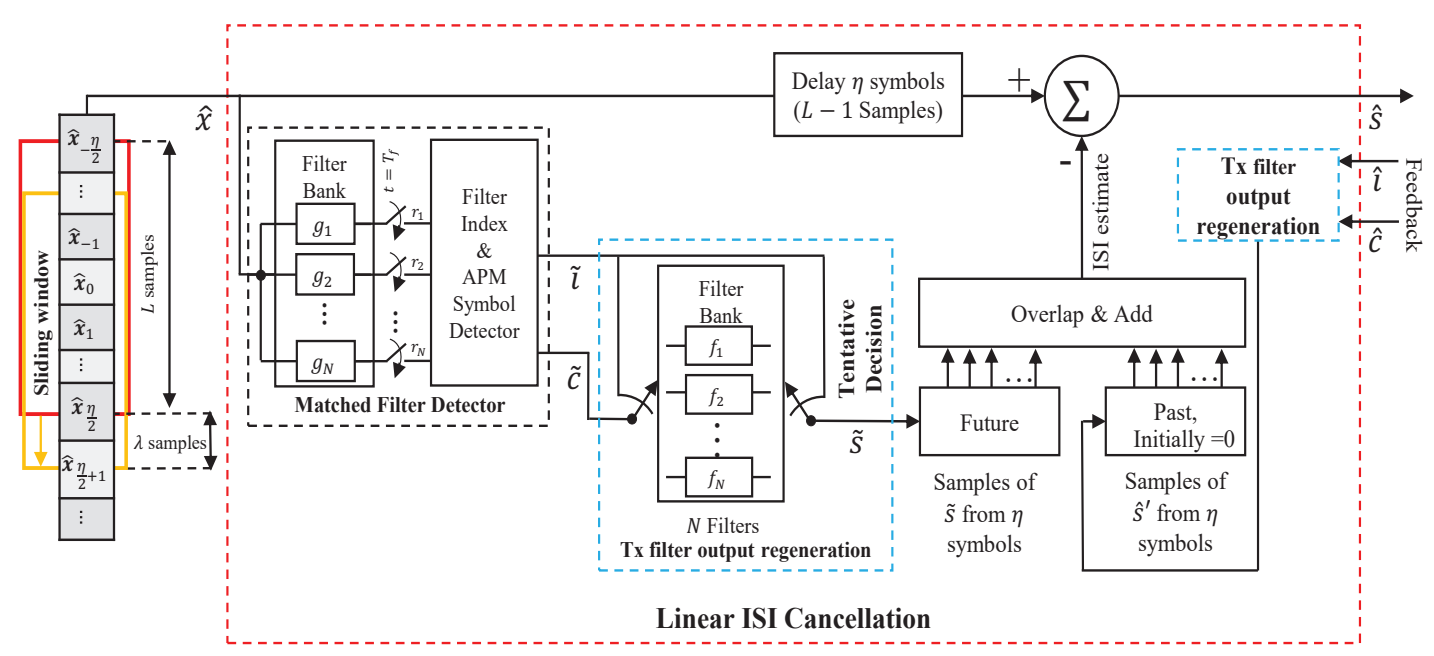

Fig. 2: ISI estimation and cancellation using feedback decisions $\{\hat{\imath}, \hat{c}\}$ for past and tentative decisions $\{\tilde{\imath}, \tilde{c}\}$ for future symbols.

condition for zero-ISI, the received signal in the AWGN channel is ISI free $(I S I=0$ in (12)) which is not the case in the proposed system.

Thus, the perfect ISI cancellation for the $L$ samples around any symbol requires the knowledge of the filter index and APM symbols for the $\eta$ past and $\eta$ future symbols where the ISI in the last $\left(L^{\text {th }}\right)$ sample of the first APM symbol $c_{0}$ can be eliminated by knowing all the future $\eta$ symbols. The proposed ISI cancellation algorithm takes the past $\eta$ detected APM symbols $\hat{c}_{n^{\prime}<n}$ along with their filter indices $\hat{\imath}_{n^{\prime}<n}$ as feedback from the previous final decisions as shown in Fig. 1. However, similar to [14], a tentative decision based on MFbased detector is required for the $\eta$ future symbols. Thus, the ISI cancellation and detection for the current symbol will be delayed by $\eta$ symbols after receiving the $L$ samples of $c_{n}$ as depicted in Fig. 2 in order to be able to mitigate the anticausal ISI. Note that the conventional transceiver delay is $d_{\text {conv }}=\frac{\eta \cdot \lambda}{2}+\left\lfloor\frac{\lambda}{2}\right\rfloor$ samples, which corresponds to filter ramp-up delay and time needed to receive all $\lambda$ samples for first symbol. Thus, the additional FSIM system delay compared to conventional transceiver is $d_{\mathrm{FSIM}}=L+\eta \lambda-d_{\mathrm{conv}}=3 \frac{\eta \cdot \lambda}{2}+1-\left\lfloor\frac{\lambda}{2}\right\rfloor$ samples, which corresponds to the delay needed to receive the rest of the first $L$ samples and to eliminate the anticausal ISI from the $\eta$ future symbols.

The FSIM receiver with linear ISI cancellation is depicted in Fig. 2. The tentative decision of the APM symbol $\tilde{c}_{n^{\prime}>n}$ and the filter index $\tilde{\imath}_{n^{\prime}>n}$ for future $\eta$ symbols are detected by the matched filter as described in subsection II-B2 using their corresponding $L$ samples of the received signal $y_{n^{\prime}}[m]$. These tentative decisions $\left\{\tilde{\imath}_{n^{\prime}}, \tilde{c}_{n^{\prime}}\right\}$ are used to regenerate the filter bank output originally generated at the transmitter side as $\tilde{s}_{n^{\prime}}[m]=\tilde{c}_{n^{\prime}} f_{\tilde{i}_{n^{\prime}}}[m]$. Similarly, this regeneration is performed for the past symbols using the feedback decisions $\left\{\hat{\imath}_{n^{\prime}}, \hat{c}_{n^{\prime}}\right\}$ to get $\hat{s}_{n^{\prime}}[m]=\hat{c}_{n^{\prime}} f_{\hat{\imath}_{n^{\prime}}}[m]$. As shown in Fig. 2 , the regenerated signals are saved in their corresponding registers and then used to generate the ISI estimation. Finally, the ISI cancellation is performed by subtracting the estimated ISI from the corresponding $L$ samples of the received signal $y_{n^{\prime}}[m]$. This aims to generate the estimated transmitter filter output $\hat{s}_{n}[m]$ for the APM symbol $c_{n}$ that can be expressed as follows:

$$
\hat{s}_{n}[m]=c_{n} f_{i_{n}}[m]+I S I_{\text {resid }}[m]+v_{n}[m],
$$

where $m=0, \ldots L-1$. The $I S I_{\text {resid }}[m]$ represents the residual ISI that might still persist due to the non-perfect ISI cancellation. Note that if the filters are well designed, the $I S I_{\text {resid }}[m]$ approaches zero. Finally, $\hat{s}_{n}[m]$ is passed through the filter index and the APM symbol detectors to recover the bitstreams $b_{1}$ and $b_{2}$ as described in Fig. 1.

\section{RESUlts AND Discussions}

This section discusses the performance comparison of the proposed FSIM transceiver with the joint ML and MF-based detectors. Then, a comparison to an equivalent conventional transceiver ( $M$-ary QAM scheme with RRC filter) of same SE is presented. Afterward, the comparison of the FSIM to the best existing single carrier SISO-IM schemes is performed in a multipath frequency-selective fading channel.

Notably, the filter bank design of $N$ filter shapes is a challenging problem. Therefore, we devise 2 and 4 nonoptimal filter shapes to demonstrate the feasibility of the proposed scheme and illustrate the minimum performance gain and SE enhancement that can be achieved. The complete responses after the matched filtering are presented in Fig. 3a for $N=2$, and in Fig. $3 b$ for $N=4$.

The proposed system is studied at different transmission rates (between 3 and 7 bits/symbol or bit per channel use (bpcu)) by using different numbers of filter shapes and different QAM orders. The simulation parameters used are summarized in Table I.

The FSIM performance curves in Figs. 4-6 are obtained using the proposed ISI estimation and cancellation, whereas 


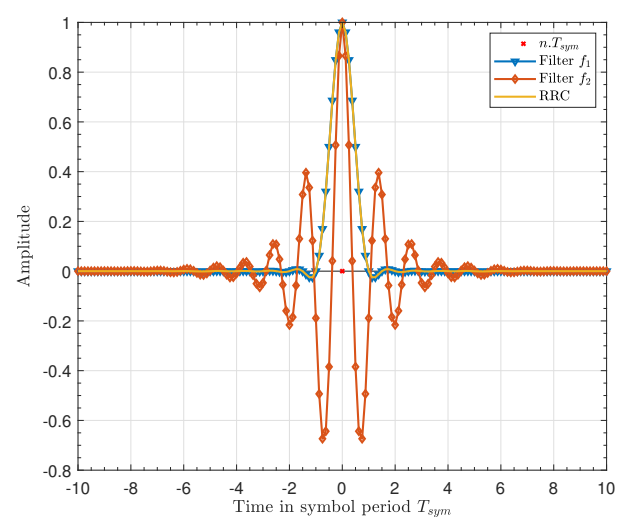

(a)

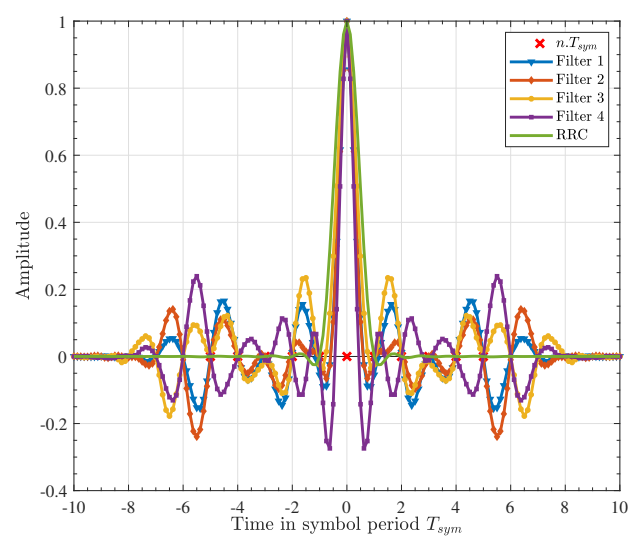

(b)

Fig. 3: Complete responses of the $N$ filters and RRC obtained after correct matched filtering at the receiver side, where $\eta=$ 10 and $\lambda=8$ : (a) $N=2$, (b) $N=4$

TABLE I: Simulation parameters

\begin{tabular}{||c|c||}
\hline Parameters & Value \\
\hline \hline$M$-ary QAM & {$[4,16,32,64]$} \\
\hline$N$ & {$[2,4]$} \\
\hline$\eta$ & 10 \\
\hline Oversampling factor: $\lambda$ & 8 \\
\hline Number of symbols & $5 \times 10^{5}$ \\
\hline pulse-shaping filter for Conventional Transceiver & Root Raised Cosine (RRC) \\
\hline
\end{tabular}

its lower bound is defined by assuming a perfect ISI estimation and cancellation. The figures 4-5 show that the performance using the joint ML and MF-based detector are very tight, but the latter has extremely low complexity.

It is clear in Figs. 4a-4b and Fig. 5 that FSIM with nonoptimal filter shapes and $M<32$ QAM achieves a significant performance gain ranging between $1 \mathrm{~dB}$ and $4 \mathrm{~dB}$ in AWGN channel at $\mathrm{BER}=10^{-4}$ compared to the equivalent conventional transceivers of same SE. In addition, these FSIM results with the proposed ISI estimation and cancellation technique approaches those with perfect ISI cancellation, especially with low order QAM, as shown in Fig. 4a and 5. However, the propagation of error issue clearly appears in the FSIM performance using the proposed ISI cancellation and high QAM order, as depicted in Fig. 4c due to the APM sensitivity to ISI and the residual ISI from the error in tentative and feedback decisions in the preceding block for ISI estimation/cancellation. Thus, the error propagation is an issue with any detector that may appear with ISI sensitive schemes if the filter bank introduces important ISI distortion, so it can be minimized or eliminated by optimal filter bank design.

In contrast to the 2-FSIM-32QAM performance in Fig. $4 \mathrm{c}$, the result using the 4 non-optimal filters with 16QAM (4-FSIM-16QAM ) achieves $2.2 \mathrm{~dB}$ gain compared to its equivalent scheme 64QAM, as depicted in Fig. 5. Thus, increasing the SE gain by filter IM domain and reducing the APM order allows to achieve better performance and EE gain, i.e. using 4-FSIM-16QAM instead of 2-FSIM-32QAM is recommended to achieve 6 bits/symbol transmission rate similar to (64QAM) but with performance gain advantage.

Figure 6 shows clearly that FSIM outperforms all other schemes with a minimum gain of $6 \mathrm{~dB}$ compared to the second-best scheme Reduced Correlation (RC) SC-TIM (RCSC-TIM) in Rayleigh selective channel with $J=2$. Note that FSIM achieves similar significant gain with a larger $J$. More details about the proposed FSIM and its theoretical performance analysis are provided in [15].

\section{CONCLUSION}

In this paper, a novel IM domain is explored by proposing a novel scheme named "Filter Shape Index Modulation". The proposed FSIM system conveys information bits by the index of filter shape and the transmitted APM symbol. It is worth mentioning that FSIM allows changing the filter shape at the symbol rate, and it exploits all available time/frequency resources. Thus, FSIM achieves higher SE gain compared to the time/frequency SISO-IM schemes. In addition, the optimal joint ML and a low complexity MF-based detector for FSIM are proposed, where the latter also achieves the optimal performance but with a prominent complexity reduction. Since the proposed FSIM scheme allows using non-Nyquist filters to maintain low filters' cross-correlation, an ISI estimation and cancellation technique is proposed. The results show that FSIM achieves a significant performance gain of up to $4 \mathrm{~dB}$ compared to the equivalent scheme of the same SE in the AWGN channel. Moreover, the FSIM superiority is also maintained in the frequency-selective Rayleigh fading channel, where it achieves a minimum gain of $6 \mathrm{~dB}$ compared to the existing single carrier SISO-IM schemes. It is worth mentioning that the proposed ISI cancellation technique allows reaching the lower-bound with perfect ISI cancellation when using low-order APM, whereas some performance degradation appears with high-order QAM due to the residual ISI and the non-optimal filter design. Finally, many possible extensions for this scheme and the challenging filter bank design will be addressed in our future work.

\section{ACKNOWLEDGMENT}

The research leading to these results received funding from the French National Research Agency (ANR-17-CE25-0013) within the frame of the project BRAVE. 


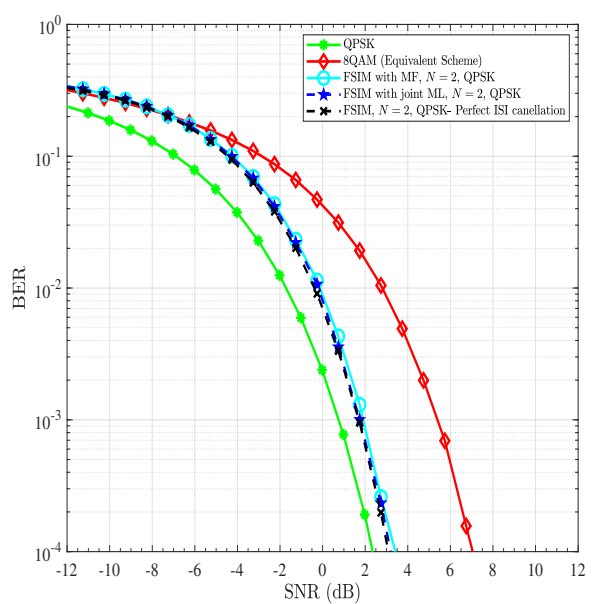

(a)

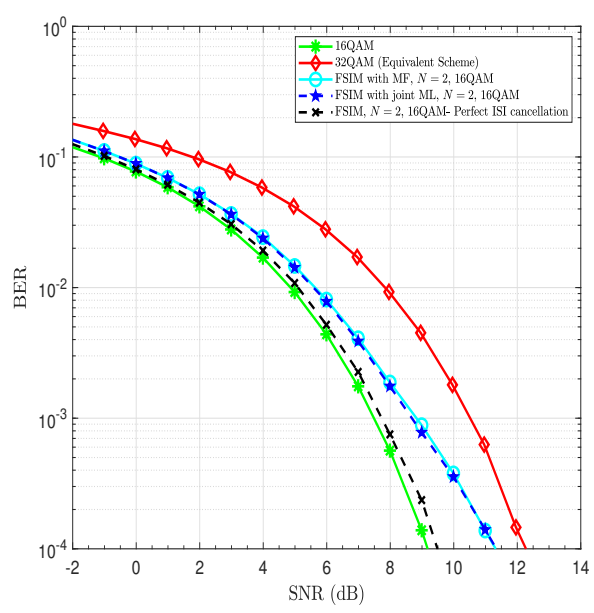

(b)

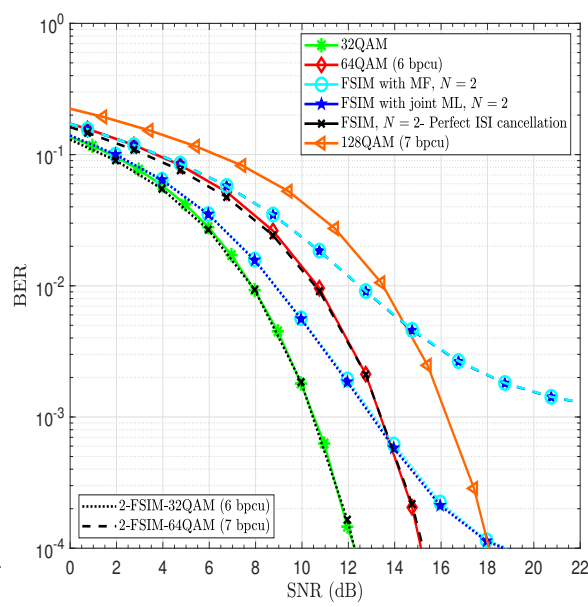

(c)

Fig. 4: BER performance of 2-FSIM-MQAM with the proposed ISI estimation/cancellation, the perfect ISI cancellation and its equivalent scheme $2 M \mathrm{QAM}$ of the same transmission rate: (a) $M=4$ (3 bits/symbol), (b) $M=16$ (5 bits/symbol), (c) $M=32$ and $M=64$ ( 6 and 7 bits/symbol).

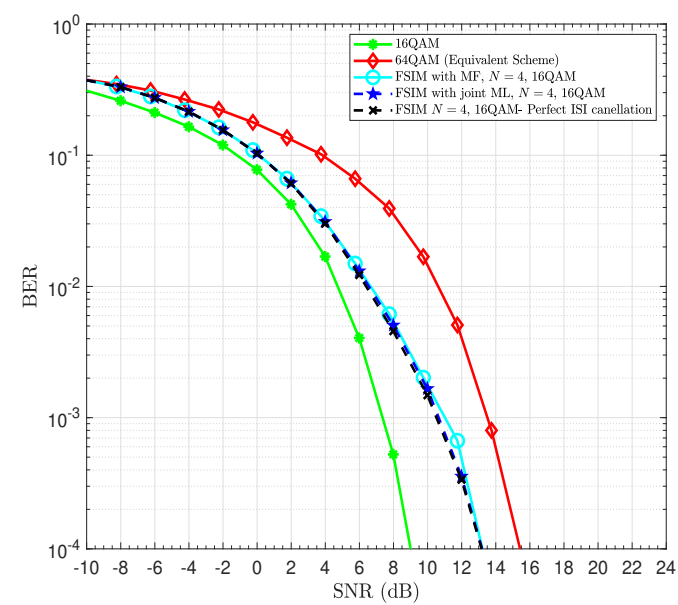

Fig. 5: BER performance of 4-FSIM-16QAM with the proposed ISI estimation/cancellation, the perfect ISI cancellation and its equivalent scheme 64QAM of the same transmission rate 6 bits/symbol.

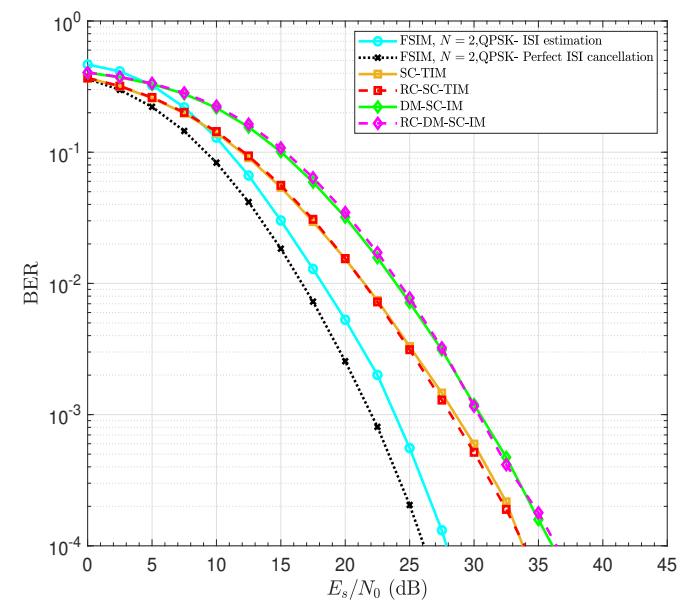

Fig. 6: Average BER comparison of different SC schemes with IM in a frequency selective Rayleigh fading channel $(\mathrm{J}=2)$.

\section{REFERENCES}

[1] J. Pierce, "Optical channels: Practical limits with photon counting," IEEE Trans. Commun., vol. COM-26, no. 12, pp. 1819-1821, Dec. 1978.

[2] M. Nakao, T. Ishihara, and S. Sugiura, "Single-carrier frequency domain equalization with index modulation," IEEE Commun. Lett., vol. 21, no. 2, pp. 298-301, Feb. 2017

[3] D. Tsonev, S. Sinanovic, and H. Haas, "Enhanced subcarrier index modulation (SIM) OFDM," in Proc. IEEE GLOBECOM Workshops, Dec. 2011, pp. 728-732

[4] E. Basar, U. Aygolu, E. Panayirci, and H. V. Poor, "Orthogonal frequency division multiplexing with index modulation," IEEE Trans. Signal Process., vol. 61, no. 22, pp. 5536-5549, 2013.

[5] R. Fan, Y. J. Yu and Y. L. Guan, "Generalization of orthogonal frequency division multiplexing with index modulation," IEEE Trans. Wireless Commun., vol. 14, no. 10, pp. 5350-5359, Oct. 2015.

[6] T. Ishihara and S. Sugiura, "Faster-than-nyquist signaling with index modulation," IEEE Wireless Commun. Lett., vol. 6, no. 5, pp. 630-633, Oct. 2017.

[7] M. Nakao and S. Sugiura, "Spectrally efficient frequency division multiplexing with index-modulated non-orthogonal subcarriers," IEEE Wireless Commun. Lett., vol. 8, no. 1, pp. 233-236, Feb. 2019.

[8] M. Nakao and S. Sugiura, "Dual-mode time-domain single-carrier index modulation with frequency-domain equalization," in 2017 IEEE 86th Veh. Technol. Conf. (VTC-Fall), Toronto, Ontario, 2017, pp. 1-5.

[9] T. Mao, Z. Wang, Q. Wang, S. Chen and L. Hanzo, "Dual-mode index modulation aided OFDM," IEEE Access, vol. 5, pp. 50-60, 2017.

[10] M. Saad, F. Bader, J. Palicot, A. C. Al Ghouwayel and H. Hijazi, "Single Carrier with Index Modulation for Low Power Terabit Systems," 2019 IEEE Wireless Communications and Networking Conference (WCNC), Marrakesh, Morocco, Apr. 2019, pp. 1-7.

[11] M. Saad, A. C. Al Ghouwayel, H. Hijazi, F. Bader and J. Palicot, "MIMO Techniques for Wireless Terabits Systems under Sub-THz Channel with RF Impairments," 2020 IEEE International Conference on Communications, Dublin, Ireland, 2020, pp. 1-6.

[12] M. Saad, F. C. Lteif, A. C. Al Ghouwayel, H. Hijazi, J. Palicot and F. Bader, "Generalized Spatial Modulation in Highly Correlated Channels," 2019 IEEE 30th Int. Symposium on Personal, Indoor and Mobile Radio Communications (PIMRC), Istanbul, Turkey, Sept. 2019, pp. 1-6.

[13] F. Pancaldi, G. M. Vitetta, R. Kalbasi, N. Al-Dhahir, M. Uysal and H. Mheidat, "Single-carrier frequency domain equalization," in IEEE Signal Process. Mag., vol. 25, no. 5, pp. 37-56, September 2008.

[14] K. Berberidis and P. Karaivazoglou, "A block adaptive DFE in the frequency domain based on tentative decisions," in 9th Eur. Signal Process. Conf. (EUSIPCO 1998), Rhodes, 1998, pp. 1-4.

[15] M. Saad, J. Palicot, F. Bader, A. C. Al Ghouwayel, and H. Hijazi, "A Novel Index Modulation Dimension based on Filter Domain: Filter Shapes Index Modulation," IEEE Transactions on Communications, vol. 69 , no. 3, 2021. doi: 10.1109/TCOMM.2020.3039842 